\title{
Remarks on Closure and Interior Operators in Bitopological Spaces
}

\author{
Thamizharasi $G^{1}$ and $P$ Thangavelu2* \\ 1 Department of Mathematics, RMD Engineering College, Chennai-601 206, India \\ 2 Department of Mathematics, Aditanar College, Tiruchendur-6282 16, India \\ E-mail: ptvelu12@gmail.com
}

Received January 20, 2010 / Accepted April 30, 2010

\begin{abstract}
Andrijevic and his collaborators studied the various aspects of closure and interior operators in general topological spaces and obtained several relations among them. Researchers in general topology studied such operators in bitopological settings. Andrijevic established that the result $\alpha c l A=A \cup c l(i n t(c \mid A))$ holds for any subset $A$ of a topological space where $\alpha c l A$, clA and int $A$ denote the $\alpha$-closure of $A$, closure of $A$ and interior of $A$ respectively. He also established the analog results for other operators in terms of the closure and interior operators in general topological spaces. In this paper we discuss the analog results in bitopological settings.
\end{abstract}

Keywords: ij-semi-closure, ii-semi-interior, $\alpha$-closure, bitopology. 


\section{Introduction}

The study of bitopological spaces was first initiated by Kelly in 1963. A large number of papers have been done to generalize the topological concepts to bitopological setting. The closure and interior operators are the two important concepts in the study of general topological structures. These two operators are widely used in computer science applications especially in Rough set theory and in Fuzzy set theory. More over the closure and interior operators are very close to the upper and lower approximations in rough set theory respectively. The existence of two topologies on a non empty set $X$ provides information about the set $X$ in two different methods. The bitopological spaces have applications in data mining using rough sets. This article dealt with the closure and interior operators in bitopological settings. Andrijevic studied the relations among the operators' in general topological spaces. Maheshwari and Prasad [5], Jelic [3], Khedr et al. [4] and Sampath Kumar [6] studied the operator's namely semi-closure, pre-closure, semi-pre-closure and $\alpha$-closure in bitopological settings. Andrijevic established the result $\alpha c / A=A \cup c /(i n t(c / A))$ for any subset $A$ of a topological space $(X, \tau)$ where clA and int $A$ denotes the closure of $A$ and interior of A respectively in $(X, \tau)$. He also established the similar results for other operators. In this paper we extend these results to bitopological spaces. In section 2 we recall the definitions of ii-operators in bitopological spaces and we study the relations among them in section 3. The symbol $\square$ indicates the end of the proof. We use the notation cl int clA for $c l(\operatorname{int}(\mathrm{cl} A))$.

\section{Preliminaries}

Throughout the paper the ordered triplet $\left(X, \tau_{1}, \tau_{2}\right)$ is a bitopological space where $\tau_{1}$ and $\tau_{2}$ are topologies on $X$. Let $A$ and $B$ be subsets of the bitopological space $\left(X, \tau_{1}, \tau_{2}\right)$.

The notations $j$-clA and $j$-intA denote the closure of $A$ and interior of $A$ with respect to the topology $\tau_{i}$ respectively. $X \backslash A$ denotes the complement of $A$ in $X$. The following definitions will be useful.

Definition 2.1. A is called

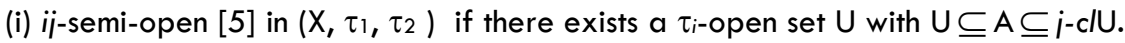

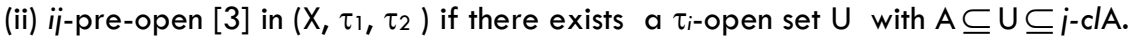

(iii) ii-semi-pre-open or ii- $\beta$-open $[4]$ in $\left(X, \tau_{1}, \tau_{2}\right)$ if there exists an ij-pre-open set $U$ in $\left(X, \tau_{1}, \tau_{2}\right)$ with $U \subseteq A \subseteq i$-clU that is if $A \subseteq i-c l(i-i n t(j-c l A))$

(iv) ij- $\alpha$-open [3], [6] in $\left(X, \tau_{1}, \tau_{2}\right)$ if $A \subseteq i$-int $(i$-cl $(i-i n t A))$.

The complement of an ij-semi-open set is ij-semi-closed. The ii-pre-closed sets, ii-semi-preclosed sets and $i j-\alpha$-closed sets will be analogously defined.

2 Published by KBM Scientific Publishing, LP (www.kbm-scientific-publishing.org) 
Maheshwari and Prasad [5] established the following theorem.

Theorem 2.2. The following results hold in a bitopological space.

(i) The union of an arbitrarily collection of ij-semi-open sets is ij-semi-open;

(ii) The intersection of two ij-semi-open sets is not ij-semi-open;

(iii) The intersection of an arbitrarily collection of ij-semi-closed sets is ij-semi-closed and

(iv) The union of two ij-semi-closed sets is not ij-semi-closed.

Jelic [3], Khedr et al. [4] and Sampath Kumar[6] respectively characterized ii-pre-open sets, iisemi-pre-open sets and $i j-\alpha$-open sets. The intersection of all ij-semi-closed sub sets of $\left(X, \tau_{1}, \tau_{2}\right)$ containing a subset $A$ of $X$ is the ij-semi-closure of $A$, denoted by ij-sclA. The union of all ij-semiopen sets contained in $A$ is called the ij-semi-interior of $A$, denoted by ij-sintA. The ij-pre-closure, ijsemi-pre-closure, ij- $\alpha$-closure, ij-pre-interior, ij-semi-pre-interior and $i j$ - $\alpha$-interior will be analogously defined respectively denoted by ii-pclA, ij-spclA, ij- $\alpha$ clA, ij-pintA, ij-spintA and ij$\alpha \operatorname{intA}$.

\section{Properties}

Andrijevic [2] established the following results for any subset A of a general topological spaces.

(i) $\quad s c / A=A \cup$ int $c l A$.

(ii) $\quad \operatorname{sint} \mathrm{A}=\mathrm{A} \cap \mathrm{clint} \mathrm{A}$.

(iii) $\quad \alpha c l A=A \cup c l$ int $c l A$.

(iv) $\quad \alpha \operatorname{int} \mathrm{A}=\mathrm{A} \cap \operatorname{int} \mathrm{cl} \operatorname{int} \mathrm{A}$.

We extend the above results to bitopological spaces.

Proposition 3.1. For any subset $A$ of $\left(X, \tau_{1}, \tau_{2}\right), A \cap i$-cl(i-intA $)$ is ii-semi-open and $A \cup i$-int $(i$-clA $)$ is ij-semi-closed.

Proof. $i-c l(i-i n t(A \cap i-c l(i-i n t A)))=i-c l(i-i n t A \cap i-i n t(j-c l(i-i n t A)))$.

$$
=i-c l(i-i n t A) \text {. }
$$

$A \cap i-c l(i-i n t A)=A \cap i-c l(i-i n t(A \cap i-c l(i-i n t A)))$

$$
\subseteq i-c l(i-i n t(A \cap i-c l(i-i n t A))) .
$$

$A \cap i$-cl(i-intA) is ij-semi-open.

This implies that $X \backslash(A \cup i$-int $(i$-clA $))=(X \backslash A) \cap i$-cl(i-int $(X \backslash A))$ is ij-semi-open. This proves that $A \cup$ $i$-int $(i$-clA $)$ is ii-semi-closed. 
Proposition 3.2. Let $A$ be a subset of $\left(X, \tau_{1}, \tau_{2}\right)$. Then $i i-\operatorname{sint} A=A \cap i-c l(i-\operatorname{int} A)$.

Proof. Let $B=i j-\operatorname{sint} A=$ the union of all ij-semi-open sets contained in $A$. $B$ is ij-semi-open and $B$ $\subseteq A$. Since $B$ is $i j$-semi-open, $B \subseteq i$-cl(i-int $B) \subseteq i$-cl(i-intA). This implies that $B \subseteq A \cap i$-cl(i-intA). Now using Proposition 3.1, $\mathrm{A} \cap i$-cl(i-intA) is ij-semi-open.

Since $A \cap i-c l(i-\operatorname{int} A) \subseteq A$, by the definition of $i j-\operatorname{sint} A, A \cap i-c l(i-i n t A) \subseteq B$ and that shows ij-sint $A=$ $A \cap i$-cl(i-intA).

Proposition 3.3. Let $A$ be a subset of $\left(X, \tau_{1}, \tau_{2}\right)$. Then ij-sclA $=A \cup i$-int $(i-c l A)$.

Proof. $\quad i j-s c l A=$ intersection of all ij-semi-closed sets of $X$ containing $A$

$$
\begin{aligned}
& =X \backslash i i-\operatorname{sint}(X \backslash A), \\
& =X \backslash((X \backslash A) \cap i-c l(i-i n t(X \backslash A)) \text {, using Proposition } 3.2 \\
& =X \backslash((X \backslash A) \cap(X \backslash i-i n t(i-c \mid A)) \\
& =A \cup i \text {-int }(i-c \mid A) .
\end{aligned}
$$

Proposition 3.4. i-int $(j-c l(i-i n t A)) \subseteq i i-s c l(i j-\operatorname{sint} A)$.

Proof. $i i-s c l(i j-\sin t A)=i i-s c l(A \cap i-c l(i-\operatorname{int} A))$, using Proposition 3.2

$$
\begin{aligned}
& =(A \cap i-c l(i-i n t A)) \cup i \text {-int }(i-c l(A \cap i-c l(i-i n t A))) \text {, using Proposition } 3.3 \\
& \supseteq(A \cap i-i n t(i-c l(i-i n t A))) \cup i-i n t(j-c l(i-i n t A \cap i-c l(i-i n t A))) \\
& =(A \cap i-i n t(j-c l(i-i n t A))) \cup i \text {-int }(i-c l(i-i n t A)) \\
& =i-\operatorname{int}(j-c l(i-\operatorname{int} A)) .
\end{aligned}
$$

Lemma 3.5. For any sub set $A$ of $\left(X, \tau_{1}, \tau_{2}\right), A \cap i$-int $(j-c l(i-i n t A))$ is $i j-\alpha$-open and $A \cup i$-cl(j-int( $i$ clA)) is $i j-\alpha$-closed.

Proof. $i-i n t(i-c l(i-i n t(A \cap i-i n t(j-c l(i-i n t A)))))=i-i n t(j-c l(i-i n t A \cap i-i n t(j-c l(i-i n t A))))$

$$
=i-\operatorname{int}(j-c l(i-\operatorname{int} A)) \text {. This implies that }
$$

$A \cap i-\operatorname{int}(j-c l(i-\operatorname{int} A))=A \cap i-\operatorname{int}(j-c l(i-\operatorname{int}(A \cap i-i n t(j-c l(i-i n t A)))))$

$$
\subseteq i-\operatorname{int}(i-c l(i-i n t(A \cap i-i n t(i-c l(i-i n t A)))))
$$

$A \cap i$-int( $j$-cl(i-intA))is ij- $\alpha$-open. This implies

$X \backslash(A \cup i-c l(j-i n t(i-c l A)))=(X \backslash A) \cap i-i n t(j-c l(i-i n t(X \backslash A)))$

$$
=i j \text { - } \alpha \text {-open that further implies }
$$

$A \cup i$-cl(j-int $(i-c \mid A))$ is $i j-\alpha$-closed.

Proposition 3.6. ii- $\alpha$ int $A=A \cap i-i n t(j-c l(i-i n t A))$.

Proof. Let $B=i j$ - $\alpha$ int $A=$ the union of all ij- $\alpha$-open sets contained in $A$.

Clearly $B$ is $i j-\alpha$-open and $B \subseteq A$. Since $B$ is $i j$ - $\alpha$-open,

$B \subseteq i-\operatorname{int}(j-c l(i-i n t B)) \subseteq i-\operatorname{int}(j-c l(i-i n t A))$. This proves that 
$\mathrm{B} \subseteq \mathrm{A} \cap i-\operatorname{int}(j-c l(i-\operatorname{int} \mathrm{A}))$. Now using Lemma 3.5,

$A \cap i-i n t(j-c l(i-i n t A))$ is $i j-\alpha$-open. By the definition of $i j-\alpha i n t A$,

$A \cap i-i n t(i-c l(i-i n t A)) \subseteq B$. Then it follows that $B=A \cap i-i n t(i-c l(i-i n t A))$.

Therefore $i i-\alpha \operatorname{int} A=A \cap i-i n t(j-c l(i-\operatorname{int} A))$.

Proposition 3.7. ii- $\alpha \mathrm{cl} A=\mathrm{A} \cup i-c l(i-i n t(i-c / A))$.

Proof. $i j-\alpha c l A=$ intersection of all $i j-\alpha$-closed sub sets of $X$ containing $A$

$=\mathrm{X} \backslash i i-\operatorname{\alpha int}(\mathrm{X} \backslash \mathrm{A})$,

$=X \backslash((X \backslash A) \cap i$-int $(j-c l(i-i n t(X \backslash A))))$, using Proposition 3.6

$=X \backslash((X \backslash A) \cap(X \backslash i-c l(j-i n t(i-c l A))))$

$=A \cup i-c l(i-i n t(i-c \mid A))$.

Andrijevic[2] also established that $\operatorname{\alpha int}(\alpha \mathrm{cl} / \mathrm{A})=\operatorname{int} \mathrm{clA}$ and

$\alpha c l(\alpha \operatorname{int} A)=c l$ int $\mathrm{A}$ in unital topological spaces. However the corresponding results in bitopological spaces do not hold as shown below.

Proposition 3.8. ii- $\alpha \operatorname{int}(j i-\alpha c \mid A) \supseteq i-i n t(j-c \mid A)$.

Proof. Using Proposition 3.6 and Proposition 3.7 we get

$i j-\operatorname{\alpha int}(j i-\alpha c \mid A)=i j-\operatorname{\alpha int}(A \cup i-c l(i-i n t(j-c \mid A)))$

$$
=(A \cup i-c l(i-i n t(j-c \mid A))) \cap i-i n t(i-c l(i-i n t(A \cup i-c l(i-i n t(j-c l A)))))
$$$$
\supseteq(A \cup i-c l(i-i n t(j-c l A))) \cap i-i n t(j-c l(i-i n t A \cup i-i n t(j-c l(i-i n t(j-c l A))))
$$$$
=(A \cup i \text {-cl(i-int }(j-c \mid A))) \cap i-i n t(i-c l(i-i n t A \cup i-i n t(j-c \mid A)))
$$$$
=(\mathrm{A} \cup j-c l(i-i n t(j-c \mid A))) \cap i-\operatorname{int}(j-c l(i-i n t(j-c \mid A)))
$$$$
=(\mathrm{A} \cup j-\mathrm{cl}(i-\operatorname{int}(j-\mathrm{cl} \mathrm{A}))) \cap i-i n t(i-c \mid \mathrm{A})
$$

$=(A \cap i-i n t(j-c \mid A)) \cup(i-c l(i-i n t(j-c \mid A)) \cap i-i n t(j-c \mid A))$

$$
=(\mathrm{A} \cap i-i n t(j-\mathrm{clA})) \cup i-\operatorname{int}(j-\mathrm{clA})
$$

$=i-\operatorname{int}(j-c \mid \mathrm{A})$.

Proposition 3.9. ij- $\alpha c l(j i-\alpha i n t A) \subseteq i-c l(j-i n t A)$.

Proof. $X \backslash i j-\alpha c l(j i-\alpha \operatorname{int} A)=i j-\alpha \operatorname{int}(X \backslash(j i-\alpha \operatorname{int} A))$,

$$
\begin{aligned}
& =i i-\operatorname{\alpha int}(j i-\alpha c l(X \backslash A)), \\
& \supseteq i-\operatorname{int}(j-c l(X \backslash A)) \text {, by Proposition } 3.8 \\
& =X \backslash i-c l(j-i n t A)
\end{aligned}
$$


This proves that $i j-\alpha c l(i i-\alpha i n t A) \subseteq i-c l(i-i n t A)$.

Proposition 3.10. ji- $\alpha \mathrm{cl}(i i-\alpha \operatorname{int}(j i-\alpha c \mid A)) \supseteq i-c l(i-i n t(j-c \mid A))$.

Proof. $j i-\alpha c l(i i-\alpha \operatorname{int}(j i-\alpha c \mid A)) \supseteq j i-\alpha c l(i-i n t(j-c \mid A))$, by Proposition 3.8

$$
\begin{aligned}
& =(i-i n t(j-c \mid A)) \cup i-c l(i-i n t(j-c l(i-i n t(j-c \mid A)))) \text {, using Proposition } 3.7 \\
& =i-\operatorname{int}(j-c \mid A) \cup i-c l(i-i n t(j-c \mid A)) \\
& =i-c l(i-\operatorname{int}(j-c \mid A)) .
\end{aligned}
$$

Proposition 3.11. ii- $\alpha \operatorname{int}(j i-\alpha c l(i j-\alpha \operatorname{int} A)) \subseteq i-i n t(j-c l(i-i n t A))$.

Proof. ii- $\alpha \operatorname{int}(j i-\alpha c l(i j-\alpha i n t A)) \subseteq i j-\alpha \operatorname{int}(j-c l(i-i n t A))$, using Proposition 3.9

$$
\begin{aligned}
& =(i-c l(i-i n t A)) \cap i-\operatorname{int}(i-c l(i-\operatorname{int}(j-c l(i-\operatorname{int} A)))) \\
& =i-c l(i-\operatorname{int} A) \cap i-\operatorname{int}(j-c l(i-\operatorname{int} A)) \\
& =i-\operatorname{int}(j-c l(i-\operatorname{int} A)) .
\end{aligned}
$$

It is observed that $\mathrm{A} \cap i$-int $(j-\mathrm{clA})$ is not $i j$-pre-open as shown below.

Example 3.12. Let $X=Z$, the set of all integers. Let $\tau_{1}=\left\{\varnothing,\{0,2\}, Z\right.$ \}and $\tau_{2}$ be the digital topology[7]. Let $A=\{0,1\} .2-c \mid A=\{0,1,2\}$ and $1-\operatorname{int}(2-c \mid A)=\{0,2\}$.

Let $B=A \cap 1-\operatorname{int}(2-c \mid A)=\{0,1\} \cap\{0,2\}=\{0\}$. Since $1-\operatorname{int}(2-c / B)=1-\operatorname{int}\{0\}=\varnothing, B$ is not 12-pre-

open. As $A \cap i$-int $(j-c \mid A)$ is not ij-pre-open, the result ii-pintA $=A \cap i$-int $(j-c \mid A)$

does not hold.

However the following proposition holds.

Proposition 3.13. ii-pint $\mathrm{A} \subseteq \mathrm{A} \cap i$-int $(j-c \mid \mathrm{A})$.

Proof. Let $B=i j-p i n t A=$ the union of all ij-pre-open sets contained in $A$

$B$ is $i j$-pre-open and $B \subseteq A$. Since $B$ is ij-pre-open, $B \subseteq i$-int $(j-c \mid B) \subseteq i$-int $(j$-clA $)$. This proves that $\mathrm{B} \subseteq \mathrm{A} \cap i-\operatorname{int}(j-\mathrm{cl} \mathrm{A})$

Proposition 3.14. ij-pclA $\supseteq A \cup i-c l(j-$ int $A)$.

\section{Proof.}

ii-pclA = X\ii-pint(X\A),

$$
\begin{aligned}
& \supseteq X \backslash((X \backslash A) \cap i \text {-int }(i-c l((X \backslash A)) \text {, using Proposition } 3.13 \\
& =X \backslash((X \backslash A) \cap(X \backslash i \text {-cl }(i-i n t A))) \\
& =A \cup i \text {-cl(j-intA). }
\end{aligned}
$$

Proposition 3.15. If $A$ is ii-semi-open then $A \cap i$-int $(j-c \mid A)$ is ij-pre-open.

Proof. i-int $(j-\mathrm{cl}(\mathrm{A} \cap i-i n t(i-c \mid A))) \supseteq i-i n t(j-c l(i-i n t A \cap i-i n t(j-c \mid A)))$

$$
=i-\operatorname{int}(i-c l(i-i n t A)) \text {. }
$$

Since $A$ is ii-semi-open, $i$-clA $=i$-cl(i-intA $)$ so that 
$i-\operatorname{int}(j-c \mid A)=i-\operatorname{int}(j-c l(i-i n t A))$. Therefore

$A \cap i-i n t(j-c \mid A)=A \cap i-i n t(j-c l(i-i n t A)) \subseteq i-i n t(j-c l(i-i n t A))$

$$
\subseteq i-\operatorname{int}(j-c l(A \cap i-i n t(j-c \mid A))) \text {. }
$$

$\mathrm{A} \cap i$-int $(j-\mathrm{clA})$ is ij-pre-open.

Corollary 3.16. If $A$ is ij-semi-closed then $A \cup i$-cl( $(-i n t A)$ is ij-pre-closed.

Proof. Suppose $A$ is ij-semi-closed. Then $X \backslash A$ is ii-semi-open. Therefore, using

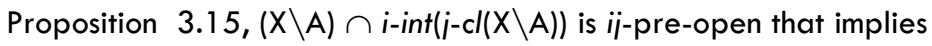

$X \backslash(A \cup i$-cl(i-intA $))$ is ii-pre-open. This proves that $A \cup i$-cl( $i$-int $A)$ is ii-pre-closed.

It is observed that $A \cap i-c l(i-i n t(j-c \mid A))$ is not $i j-$-semi-pre-open as shown below.

Example 3.17. Let $X=Z$, the set of all integers. Let $\tau_{1}=\{\varnothing,\{0,2\}$, Z $\}$ and $\tau_{2}$ be the digital topology[7]. Let $A=\{1,2\} .2-c l A=\{0,1,2\}$ and $2-c l(1-\operatorname{int}(2-c \mid A))=2-c l\{0,2\}=\{0,2\}$. Let $B=A$ $\cap 2-c l(1-\operatorname{int}(2-c \mid A))=\{1,2\} \cap\{0,2\}=\{2\}$.

Since $2-c l(1-\operatorname{int}(2-c / B))=2-c l(1-\operatorname{int}\{2\})=2-c l \varnothing=\varnothing, B$ is not 12 -semi-pre-open.

Proposition 3.18. ii-spint $A \subseteq A \cap i-c l(i-i n t(j-c \mid A))$.

Proof. Let $\mathrm{B}=$ ij-spintA

$$
=\text { the union of all ij-semi-pre-open sets of } X \text { contained in } A \text {. }
$$

$B$ is $i j$-semi-pre-open and $B \subseteq A$. Since $B$ is $i j$-semi-pre-open,

$\mathrm{B} \subseteq i-\mathrm{cl}(i-\operatorname{int}(j-\mathrm{clB})) \subseteq i-\mathrm{cl}(i-i n t(j-\mathrm{clA}))$.

Proposition 3.19. ij-spclA $\supseteq A \cup i$-int(i-cl(j-intA $))$.

Proof. ij-spclA $=X \backslash i j$-spint $(X \backslash A)$,

$$
\begin{aligned}
& \supseteq X \backslash((X \backslash A) \cap i-c l(i-i n t(j-c l(X \backslash A)))) \text {, using Proposition } 3.18 \\
& =X \backslash((X \backslash A) \cap(X \backslash i-i n t(i-c l(j-i n t A))) \\
& =A \cup i-i n t(i-c l(j-i n t A)) .
\end{aligned}
$$

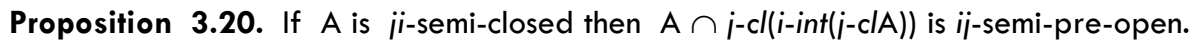

Proof. Let $B=A \cap i-c l(i-i n t(j-c \mid A))$.

$i-c l(i-i n t(j-c \mid B)) \supseteq i-c l(i-i n t(j-c l(i-i n t A \cap i-c l(i-i n t(j-c \mid A))))$

$$
\begin{aligned}
& =i-c l(i-i n t(i-c l(i-i n t A))) \\
& =i-c l(i-i n t A)) .
\end{aligned}
$$

Since $A$ is $i i$-semi-closed, $i$-int $A=i$-int $(j-c \mid A)$ so that

$$
\begin{aligned}
& i \text {-cl }(i-i n t A))=i-c l(i-i n t(j-c \mid A)) . \text { Therefore } B=A \cap i-c l(i-i n t(j-c \mid A)) \\
& =\mathrm{A} \cap(i-c l(i-i n t A)) \subseteq i-c l(i-i n t A) \\
& \subseteq i-c l(i-i n t(j-c / B)) \quad \text { by }(*)
\end{aligned}
$$

This proves that $A \cap i-c l(i-\operatorname{int}(j-c \mid A))=B$ is $i j$-semi-pre-open. 
Corollary 3.21. If $\mathrm{A}$ is $i i$-semi-open then $\mathrm{A} \cup i$-int $(i-c l(j-i n t A))$ is ij-semi-pre-closed.

Proof. Suppose $A$ is $i i$-semi-open. Then $X \backslash A$ is $i i$-semi-closed.

So, using Proposition 3.20, $(X \backslash A) \cap i$-cl $(i$-int $(i-c l(X \backslash A)))$ is ij-semi-pre-open that implies $X \backslash(A \cup i$ $\operatorname{int}(i$-cl( $(j-$ intA $)))$ is ij-semi-pre-open.

This proves that $A \cup i$-int $(i$-cl $(j-$ int $A))$ is ii-semi-pre-closed.

\section{References}

[1] Andrijevic D (1984) Some properties of the topology of $\alpha$-sets. Mat. Vesnik 36: 1-10.

[2] Andrijevic D (1986) Semi-pre-open sets. Mat. Vesnik 38: 24-32.

[3] Jelic M (1990) A decomposition of pairwise continuity. J.Inst. Math. Comput. Sci. Math.Ser.3: 25-29.

[4] Khedr FH, SM Al Areefi and T Noiri (1992) Precontinuity and semi-precontinuity in bitopological spaces. Indian J. pure appl. Math. 23(9): 625-633.

[5] Maheshwari SN and R. Prasad (1977/78) Semi-open sets and semi-continuous functions in bitopological spaces. Math. Notae 26: 29-37.

[6] Sampath Kumar S (1997) On a decomposition of pairwise continuity. Bull. Cal. Math. Soc. 89: $441-446$.

[7] Thangavelu P (2007) On the subspace topologies of the Khalimsky topology, Proc.Second International Conference on Mathematics: Trends and Developments, The Egyptian Mathematical Society, Cairo, 27-30, Dec.2007 Vol (III) Top.\& Geom.: 157-168. 